\title{
Cobalt oxide-Carbon nanocatalysts with highly enhanced catalytic performance for the green synthesis of nitrogen heterocycles through Friedländer condensation
}

\author{
Marina Godino-Ojer, a,b Antonio J. López Peinado, a Francisco J. Maldonado-Hódar, ${ }^{c *}$ Esther Bailón-

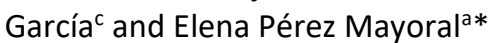

\begin{abstract}
a. Departamento de Química Inorgánica y Química Técnica, Universidad Nacional de Educación a Distancia, UNED, Paseo Senda del Rey 9, Facultad de Ciencias, 28040, (Madrid, Spain).

b. Facultad de Ciencias Experimentales, Universidad Francisco de Vitoria, UFV, Ctra. Pozuelo-Majadahonda km 1.800, 28223, Pozuelo de Alarcón (Madrid, Spain).

c. Departamento de Química Inorgánica, Universidad de Granada, UGR, Facultad de Ciencias, 18071, (Granada, Spain)

Novel series of eco-sustainable catalysts developed by supporting CoO-nanoparticles on different carbon supports, highly efficient in the synthesis quinolines and naphthyridines through the Friedländer condensation, are reported for the first time. Textural properties, dispersion and location of the Co-phase are influenced by the nature of carbon support, Coprecursor salt, and metal loading, having an important impact on the catalytic performance. Thus, the presence of the mesoand macropores in carbon aerogels together the homogenous distribution of the active phase favours the formation of the product $3 a$ as function of the metal loading. However, the increase of the metal content when using CNTs implies the formation of $\mathrm{CoO}$ aggregates and an optimal concentration of $3 \%$ wt $\mathrm{CoO}$ was observed providing the highest conversion values. The carbon-based catalysts herein reported can be considered a sustainable alternative showing advantages such as easy preparation, superior stability and notably enhanced catalytic performance, operating at lower temperature and under solvent-free conditions.
\end{abstract}

\section{Introduction}

Development of new catalytic technologies by using carbon materials constitutes a currently hot topic. Nanocarbons as their different forms could be considered almost ideal supports for catalytic purposes due to their unique characteristics such as high surface areas, chemical inertness and thermal stability, among others, providing the appropriate chemical environment to produce fine chemicals. ${ }^{1,2}$ In this sense, in the last decades metal-supported carbon-based materials applied in catalysis have particularly attracted much attention. ${ }^{3}$ Activated carbons (ACs) have been used in catalysis for a long time. ${ }^{4}$ However, the application of nanostructured carbon forms such as carbon nanotubes (CNTs) and more recently carbon aerogels ${ }^{5}$ are issues of interest and continuously growing. For instance, $\mathrm{Pd} / \mathrm{Co}$ nanoparticles supported on multi-walled CNTs have recently reported in the microwave-assisted efficient synthesis of substituted tetrazoles ${ }^{6}, \mathrm{Cu}$ loadings on $\mathrm{Ni} /$ carbon nanotubes catalysts in the hydrodeoxygenation reaction of guaiacol 7 , and RuCNT nanohybrid in the selective catalytic reduction of nitrochalcones to quinoline $\mathrm{N}$-oxides ${ }^{8}$. AuCNTs have been also found to be interesting catalysts in diverse chemical transformations such as oxidation reaction of silanes ${ }^{9}$, alcohols $^{10}$, and phenols ${ }^{11}$, reductive amination ${ }^{12}$ deoxygenation of amine $\mathrm{N}$-oxides ${ }^{13}$, aldehydes $\mathrm{N}$-formylation ${ }^{14}$ and in the synthesis of quinoxalines ${ }^{15}$. More recently, cobalt-carbon composites have been applied in the reduction of $p$-nitrophenol and pendimethaline ${ }^{16}$ and as electrocatalysts for oxygen evolution reactions ${ }^{17}$.

As one of our research lines, we have developed different environmental-friendly and efficient methodologies for the synthesis of quinolines, through Friedländer condensation ${ }^{18}$ involving acid and basic activated carbons ${ }^{19-20}$ and much more recently metal-free carbon aerogels ${ }^{21}$ and transition metal doped carbon aerogels. ${ }^{22-23}$ As far as we know, our studies represent the first examples of transition metal doped carbon aerogels implied in the aldol-based reactions, demonstrating that $\mathrm{Co}^{0}$ or $\mathrm{Cu}^{0}$ nanoparticles over the surface on carbon aerogels are the main active catalytic species producing quinolines in high yields.

With these items in mind, the goal of this paper is aimed onto the comparative study of using three types of carbon forms in the synthesis of quinolines and related compounds, via Friedländer reaction, in which Co nanoparticles are supported. The carbon-based materials of our choice are a carbon aerogel B-500, commercial AC, Norit RX 3, and multiwall carbon nanotubes (MWCNTs) showing different morphologies and textures. While Norit RX $3 \mathrm{AC}$ is mainly a microporous sample, the B-500 aerogel shows a mesoporous character. It is noticeable that the polymerization resorcinol-formaldehyde ( $R$ F) leading to nanoestructured carbon gels allows the control of the porosity and morphology of these materials as well as of their tuned surface chemistry. In fact, the relationship of the surface properties and the catalytic behaviour of these nanocarbons are continuously revised hot topics in this field. ${ }^{5}$ In this work we seek to develop a novel sustainable series of carbon-based Co catalysts with enhanced catalytic performance. At this regard, the relationship between the morphology, textural properties of the different carbon samples and the effect of the concentration and dispersion of active phases onto the catalysts and the catalytic performance are envisioned. 


\section{Materials and Methods}

\section{Synthesis and characterization of the nanocatalysts}

Three series of Co-catalysts were prepared by impregnation of different types of carbon materials with cobalt salts. Commercial AC Norit RX3 and MWCNTs have been purchased from NORIT Nederland B.V. and Sigma-Aldrich, respectively. Norit RX3 was milled and sieved to a particle size below 0.25 $\mathrm{mm}$, without any chemical treatment, but MWCNTs were firstly treated with concentrated $\mathrm{HNO}_{3}$, under refluxing conditions, according the experimental protocol reported by Trépanier et al. ${ }^{24}$ B500 carbon aerogel was synthesized in our laboratories from resorcinol $(R)$ and formaldehyde $(F)$ polymerization. The experimental protocol for the synthesis was reported elsewhere. 5,25 In this case, resorcinol (R), formaldehyde ( $F$ ) (molar ratio $\mathrm{R} / \mathrm{F}=1: 2)$ and $\mathrm{Na}_{2} \mathrm{CO}_{3}$ (C) $(\mathrm{R} / \mathrm{C}=300)$ were dissolved in water (W) and cast into glass moulds $(25 \mathrm{~cm}$ length $x 0.5 \mathrm{~cm}$ internal diameter). After the curing period (1 day at room temperature, 2 days at $303 \mathrm{~K}$ and 5 days at $353 \mathrm{~K}$ ), the gel rods were cut in $5 \mathrm{~mm}$ pellets, exchanged with acetone and supercritically dried with carbon dioxide. B500 carbon aerogel was obtained by carbonization of this organic aerogel at $773 \mathrm{~K}$ in $\mathrm{N}_{2}$ flow in a tubular furnace using a temperature ramp of 1.5 $\mathrm{K} \mathrm{min}^{-1}$ and a soak time of $2 \mathrm{~h}$. No activating agents were applied. The final carbonized is milled and sieved also below $0.25 \mathrm{~mm}$.

The cobalt-supported catalysts were prepared by treatment of the different carbon materials with the appropriate aqueous solution of cobalt nitrate $\left(\mathrm{Co}\left(\mathrm{NO}_{3}\right)_{2}\right)$ or cobalt acetate, $\left(\left(\mathrm{CH}_{3} \mathrm{CO}_{2}\right)_{2} \mathrm{Co}\right)$, to obtain the corresponding Co-loading. Briefly, carbon supports $(5 \mathrm{~g})$ were impregnated with differently concentrated $\mathrm{Co}\left(\mathrm{NO}_{3}\right)_{2}$ water solutions $(25 \mathrm{~mL})$. The used $\mathrm{Co}\left(\mathrm{NO}_{3}\right)_{2} \cdot 6 \mathrm{H}_{2} \mathrm{O}$ amounts were $0.247,0.741$, and $1.235 \mathrm{~g}$ corresponding to 1, 3 and 5\% wt. After drying overnight at 383 $\mathrm{K}$, the samples were pre-treated in He-flow at $573 \mathrm{~K}$ to obtain the $\mathrm{Co}$ active phase. Similar protocol was performed for the $\left(\mathrm{CH}_{3} \mathrm{CO}_{2}\right)_{2} \mathrm{Co}$ impregnated samples by using the appropriated cobalt salt amounts. Three catalyst series: $\mathrm{CNT}_{\text {ox }} \mathrm{nCo}$, B500nCo and NoritnCo were obtained, where $\mathrm{n}$ is the theoretic Co loading expressed as \% wt. Samples in which the cobalt salt for impregnation was $\left(\mathrm{CH}_{3} \mathrm{CO}_{2}\right)_{2} \mathrm{Co}$ were marked using Ac notation.

The morphology of carbons was analysed using a scanning electron microscope (Leo, Carl Zeiss, Geminy-153). Images were obtained at excitation energy of $20 \mathrm{kV}$. The textural characteristics of the samples were obtained by mercury porosimetry and gases adsorption. The macro-mesoporosity of the samples was analysed by mercury porosimetry (Autoscan60 from Quantachrome). The adsorption isotherms of $\mathrm{N}_{2}$ and $\mathrm{CO}_{2}$ at 77 and $273 \mathrm{~K}$ were determined using an Autosorb 1 from Quantachrome after outgassing samples overnight at $383 \mathrm{~K}$ under high vacuum $\left(10^{-6} \mathrm{mbar}\right)$. Parameters as BET surface area and micropore volume $\left(\mathrm{W}_{0}\right)$ were calculated by applying respectively the BET or Dubinin-Radushkevich equations to the isotherms. ${ }^{26,27}$ The pore size distributions (PSD) were determined by applying Quenched Solid Density Functional Theory (QSDFT), assuming slit-shaped pores, to the $\mathrm{N}_{2}$ adsorption isotherms. ${ }^{28}$ The total pore volume $\left(V_{T}\right)$ is considered as the volume of adsorbed nitrogen at a relative pressure $\mathrm{P} / \mathrm{P}_{0}=0.95 .{ }^{29}$
Thermal treatments of samples were simulated by thermogravimetric analysis using a SHIMADZU mod. TGA-50H for TG-DTG experiments. The metal content of the supported catalysts was obtained by burning a fraction of it in the thermobalance at $1123 \mathrm{~K}$ under air flow up to constant weight. Thermal Programmed Desorption (DTP) curves of carbon monoxide, carbon dioxide, water and hydrogen were recorded. Experiments were carried out by heating $150 \mathrm{mg}$ of sample at $303 \mathrm{~K} \mathrm{~min}^{-1}$ up to $1273 \mathrm{~K}$ using He as the carrier gas $\left(60 \mathrm{~cm}^{3} \mathrm{~min}^{-}\right.$ 1). The analysis of desorbed gases was performed with a Mass Spectometer model Prisma (Pfeiffer).

The chemical nature and dispersion of the metal were studied by X-ray diffraction (XRD, Phillips PW1710 diffractometer), highresolution transmission electron microscopy (HRTEM, Phillips CM-20 or HAADF FEI TITAN G2 microscope) equipped with FEI system of EDX analysis. More than 100 metal nanoparticles were analysed in each case from the corresponding microphotographs using the appropriate software (ImageJ) where a mean diameter is calculated. X-ray photo emission spectra (XPS) were recorded using a Kratos Axis Ultra-DLD spectrometer. This instrument utilizes as the radiation source $\mathrm{Mg} \mathrm{K \alpha}(1253.6 \mathrm{eV})$ and a hemispheric electron analyser operating at $12 \mathrm{kV}$ and $10 \mathrm{~mA}$.

\section{Catalytic performance}

In a typical experiment, a mixture of the corresponding 2aminoaryl aldehyde 1 ( 0.5 or $2 \mathrm{mmol}$ ) and ethyl acetoacetate 2 ( $5 \mathrm{mmol}$ ) in a three-necked vessel, equipped with thermometer, was placed on a multiexperiment work station StarFish (Radley's Discovery Technologies IUK). When the temperature reaches 303 or $323 \mathrm{~K}$, the catalyst was added $(25 \mathrm{mg})$ and the reaction mixture was stirred during $240 \mathrm{~min}$. The samples were periodically taken at 15, 30,60,120, 180 and $240 \mathrm{~min}$, diluted with acetone $(0.5 \mathrm{~mL})$, and the catalyst was filtered off and the solvent evaporated in vacuo. The reactions were followed by TLC chromatography performed on DC-Aulofolien/Kieselgel 60 F245 (Merk) using mixtures of $\mathrm{CH}_{2} \mathrm{Cl}_{2} / \mathrm{EtOH}$ 98:2 as eluent. Product was characterized by ${ }^{1} \mathrm{H}$ NMR spectroscopy. NMR spectra were recorded by using a Bruker AVANCE DPX-300 spectrometer $\left(300 \mathrm{MHz}\right.$ for $\left.{ }^{1} \mathrm{H}\right) .{ }^{1} \mathrm{H}$ chemical shifts in $\left[\mathrm{d}_{6}\right]$ DMSO are referenced to internal tetramethylsilane. 

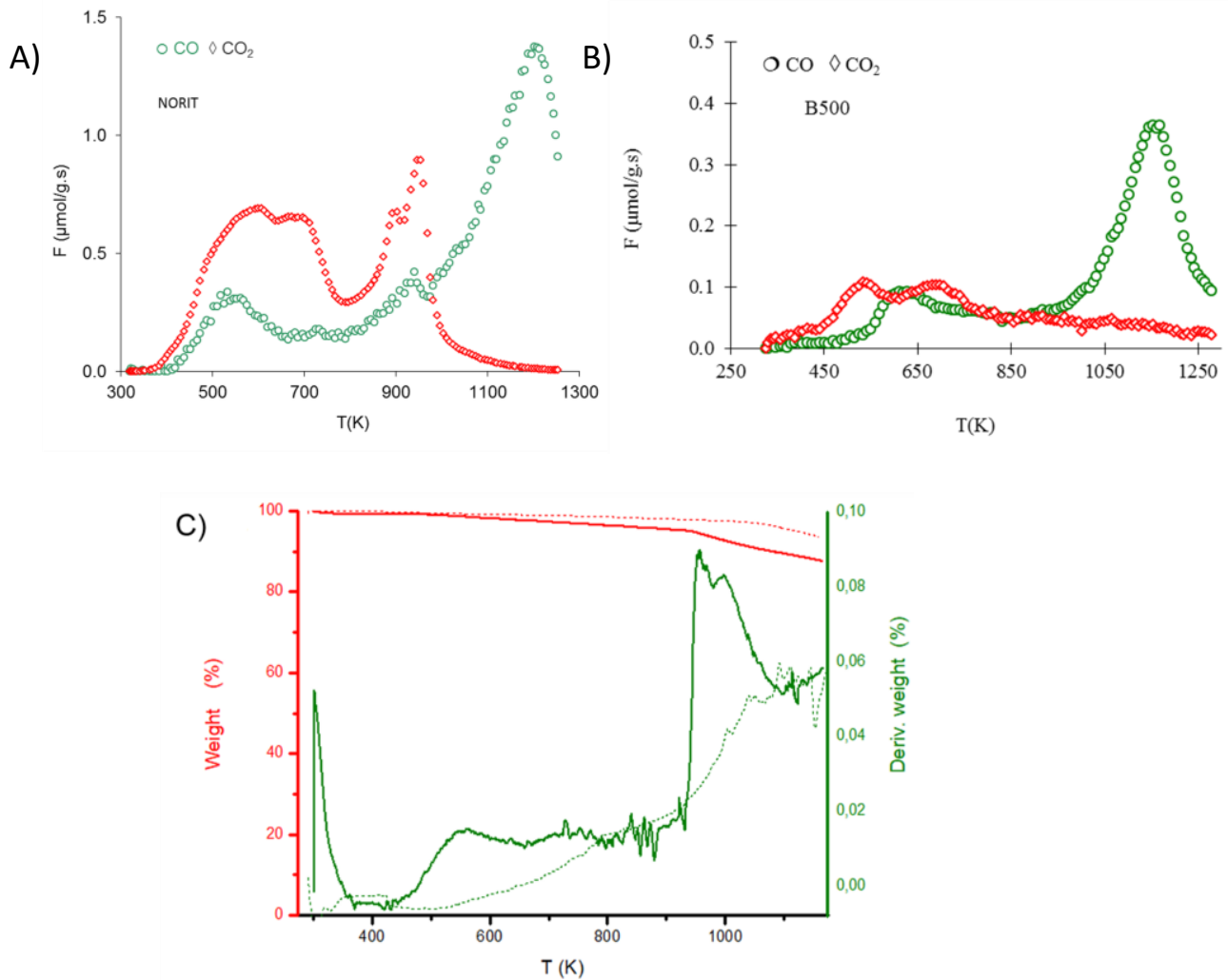

Fig. $1 \mathrm{CO}$ and $\mathrm{CO}_{2}$ DTP profiles of A) Norit and B) B500 supports C) TG-DTG profiles in He flow of CNT (---) and CNT ox (--) supports.

\section{Results and Discussion}

\section{Physico-chemical characterization of the carbon catalysts}

The physico-chemical properties of the final catalysts will be related with the characteristics of supports. Considering that the samples were prepared by impregnation, the metal content obtained by TG for the different catalysts fits according the programed loading. The elemental analysis of $\mathrm{CNT}_{\mathrm{ox}}, \mathrm{B} 500$ and Norit supports and the oxygen content calculated from the DTP experiments are collected in Table 1 . Heating in inert atmosphere the weight loss (WL) undergone by the samples corresponds to the amount of gases evolved as $\mathrm{CO}_{x}$ from the decomposition of the oxygenated surface groups (OSG), which can be also characterized as a function of their thermal stability. The higher oxygen content determined by elemental analysis for the Norit support is influenced by ash content of this sample $(4.8 \%)$. In fact, the oxygen content determined by DTP is only around $2 \%$. Lower oxygen content is determined for B500 aerogel. In this case, the only oxygen source is the resorcinolformaldehyde used for the synthesis that is forming methyleneether links between aromatic rings in the organic aerogels, but these links are mainly evolved during the carbonization process. ${ }^{30}$ This sample is obviously ash free, because it is synthesized from pure organic monomers. The corresponding
$\mathrm{CO}$ and $\mathrm{CO}_{2}$ DTP profiles are showed in Fig. $1 \mathrm{~A}$ and $1 \mathrm{~B}$. The presence of different maxima in the profile shows the presence of different types of OSG with different stability. The first peak at low temperature (at around $543 \mathrm{~K}$ ) corresponds to the evolution of carboxylic acid groups. When increasing temperature, the decomposition of OSG such as anhydrides, formed from dehydration of adjacent carboxylic groups occurs. Mainly in the case of Norit sample there are present other $\mathrm{CO}_{2}-$ evolving groups with higher thermal stability. The peak at around $973 \mathrm{~K}$ is associated to the decomposition of lactone groups. In the case of the CO-profile, $\mathrm{CO}$ is also formed from anhydride groups $\left(\mathrm{CO}+\mathrm{CO}_{2}\right)$, and with increasing temperatures from phenol, carbonyl or ether OSG, the latter decomposing at around $1173 \mathrm{~K} .{ }^{31,32}$ Similar conclusions that those previously described from TPD was pointed out when analysing the profiles obtained by TG-DTG in He flow (Fig. 1C). 
Table 1. Elemental analysis of the supports.

\begin{tabular}{|c|c|c|c|c|c|c|}
\hline Sample & $\begin{array}{l}\text { C } \\
\text { (\%) }\end{array}$ & $\begin{array}{l}\mathrm{H} \\
\text { (\%) }\end{array}$ & $\begin{array}{l}\mathrm{N} \\
(\%) \\
\end{array}$ & $\begin{array}{l}\mathrm{S} \\
\text { (\%) }\end{array}$ & $\begin{array}{l}\mathrm{O}^{[a]} \\
(\%)\end{array}$ & $\begin{array}{l}\mathrm{O}_{\text {DTP }} \\
(\%)\end{array}$ \\
\hline Norit & 89.0 & 0.3 & 0.7 & 0.0 & 11.0 & 2.8 \\
\hline B500 & 96.3 & 0.2 & 0.0 & 0.0 & 3.5 & 1.2 \\
\hline $\mathrm{CNT}_{\text {ox }}$ & 92.0 & 0.4 & 0.2 & 0.0 & 7.4 & $5.1^{[b]}$ \\
\hline CNT & 94.2 & 0.5 & 0.6 & 0.1 & 4.7 & $2.5^{[\mathrm{b}]}$ \\
\hline
\end{tabular}

experiments.

The oxygen content for $\mathrm{CNT}_{\text {ox }}$ support evidently increases after $\mathrm{HNO}_{3}$ treatment regarding raw CNT (Table 1), changing also the nature of the oxygen surface groups. Thus, it is observed that effectively the WL of $\mathrm{CNT}_{\text {ox }}>$ CNT due to the formation of OSG. The clear maximum at around $550 \mathrm{~K}$ observed for $\mathrm{CNT}_{\text {ox }}$ sample corresponding to the evolution of carboxylic acid groups while the main desorption occurring at around $973 \mathrm{~K}$ associated to the $\mathrm{CO}$ evolved from ether or carbonyl groups.

The morphology of the samples was investigated by SEM/TEM (Fig. 2). The structure of aerogel B500 corresponds to the wellknown structure built by aggregation of nearly spherical primary particles. The support $\mathrm{CNT}_{\text {ox }}$ also maintains evidently the typical morphology of MWCNT while Norit AC is a particulate material with more or less rough surface (not shown). This morphology is also related with the porosity of the samples.

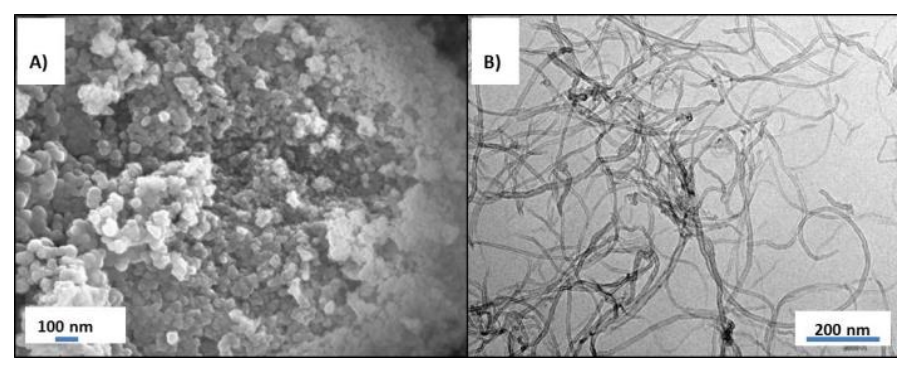

Fig. 2 Morphology of A) B500 and B) CNT ox supports.

The larger macro-mesoporosity of the samples was characterized by mercury porosimetry while the narrowest meso-microporosity was studied by gas adsorption (Fig. 3 and Table 2). The pore size distributions (PSD) obtained by mercury porosimetry (Fig. $3 \mathrm{~A}$ ) point out that carbon aerogel B500 is a mesoporous sample, with a homogenous distribution of pores with diameter around $10 \mathrm{~nm}$ whereas the Norit $A C$ is a macroporous sample with pores with diameter around $700 \mathrm{~nm}$. When analysing the $\mathrm{N}_{2}$-adsorption isotherms (Fig. $3 \mathrm{~B}$ ), it is observed that the Norit support presents however the greater microporosity pointed out by a fast increase in the adsorption capacity at low relative pressures $\left(\mathrm{P} / \mathrm{P}_{0}\right)$; the isotherm is clearly type I typical of microporous samples. However, the large knee previous to the plateau pointed out certain heterogeneity in the microporosity as well the slight slope of the plateau the existence of certain mesoporosity. In the case of B500, the adsorption isotherm is clearly type IV, corresponding to mesoporous samples, the slope of the plateau and the hysteresis cycle from $\mathrm{P} / \mathrm{P}_{0}>0.6$ denoting the presence of mesopores. The isotherm obtained for $\mathrm{CNT}_{\text {ox }}$ sample is mainly type III, denoting a weak interaction with the adsorbate. The micropore volume is quite low and the slope of the plateau and the hysteresis cycle, mainly at high relative pressures, also denotes the formation of certain mesoporosity. The application of the DFT method to analyse these isotherms allows to obtain the corresponding PSD, as showed in Fig. 3C. Results are consistent with those previously obtained by mercury porosimetry, B500 presenting again a monomodal PSD around pores of $10 \mathrm{~nm}$ of diameter, the PSD of $\mathrm{CNT}_{\text {ox }}$ being also quite similar, while in the case of the Norit AC the mesoporosity is scarce and the PSD profile is maxima for narrowest micropores. The PSD obtained for $\mathrm{CNT}_{\text {ox }}$ confirms that in general the mesoporosity corresponds to interparticle spaces formed into the CNT aggrupation, ${ }^{33}$ considering that the internal diameter is clearly smaller than the mesopore size (between 2-50 $\mathrm{nm}$ ) (see photos below).

When analysing the textural properties of the catalysts regarding their corresponding supports, it is observed that a certain blockage of the narrowest microporosity by the metal nanoparticles, but the PSD of macro and mesopores remain practically unchanged. This blockage evidently is related, therefore, with the metal loading and dispersion, which is in turn, influenced by the support and metal precursor nature as well the thermal treatment of activation. In Figure $3 D$ is showed, as an example, the influence of the precursor on the textural properties of the catalysts. Although as commented, the microporosity decreases in both cases regarding the activated carbon support, this blockage is stronger, for the same metal loading and pre-treatment, when using nitrate instead cobalt acetate. Nevertheless, both catalysts showed a similar hysteresis cycle at the same range of $\mathrm{P} / \mathrm{P}_{0}$ than those observed in the support, indicating that the mesoporosity is preserved. The textural characteristics of supports and catalyst derivatives are numerically summarized in Table 2. The study of the microporosity is also complemented by $\mathrm{CO}_{2}$ adsorption, considering that this adsorbate can be adsorbed into the narrowest microporosity, i.e. in pores with diameter $<0.7 \mathrm{~nm} .^{34}$ The higher surface values correspond to the Norit support as consequence of the high microporosity of this sample as above commented, while $\mathrm{CNT}_{\text {ox }}$ presents the lowest surface area value in spite to present the highest total pore volume, $\mathrm{V}_{\mathrm{T}}$. In all cases, the porosity of the catalysts changes regarding their supports, this feature being influenced by the location and distribution of the metallic particles, as detected in previous works. ${ }^{35}$ Thus, when supported on $\mathrm{CNT}_{\text {ox }}, \mathrm{S}_{\mathrm{BET}}$ surface area values do not change with the Co-loading, even the micropore volume determined by $\mathrm{N}_{2}$-adsorption increasing. The pore blockage in this case occurs inside the narrowest micropores, determined by $\mathrm{CO}_{2}$-adsorption $\left(\mathrm{W}_{0}\left(\mathrm{CO}_{2}\right)\right.$ which progressively decreased (Table 2). On the contrary, using Norit as support the stronger micropore blockage occurs in the larger microporosity, $\mathrm{W}_{0}$ $\left(\mathrm{N}_{2}\right)$, and is stronger when using nitrate instead acetate, suggesting a different metal nanoparticle size. In this case $\mathrm{W}_{0}\left(\mathrm{CO}_{2}\right)$ and consequently the $\mathrm{S}_{\mathrm{CO} 2}$ surface area, increases regarding the corresponding supports because the narrowest microporosity is enhanced due to the formation of metal nanoparticles inside the larger microporosity. 
Table 2. Porosity parameters of the samples.

\begin{tabular}{llllll}
\hline Sample & $\mathrm{S}_{\mathrm{BET}}\left(\mathrm{m}^{2} \mathrm{~g}^{-1}\right)$ & $\mathrm{S}_{\mathrm{CO} 2}\left(\mathrm{~m}^{2} \mathrm{~g}^{-1}\right)$ & $\mathrm{W}_{\mathrm{O}(\mathrm{N} 2)}\left(\mathrm{cm}^{3} \mathrm{~g}^{-1}\right)$ & $\mathrm{W}_{\mathrm{O}(\mathrm{CO} 2)}\left(\mathrm{cm}^{3} \mathrm{~g}^{-1}\right)$ & $\mathrm{V}_{\mathrm{T}}\left(\mathrm{cm}^{3} \mathrm{~g}^{-1}\right)$ \\
\hline $\mathrm{CNT}_{\text {ox }}$ & 339 & 121 & 0.14 & 0.10 & 2.08 \\
CNT $_{\text {ox }}$ 1Co & 219 & 65 & 0.08 & 0.05 & 1.49 \\
CNT $_{\text {ox } 3 \text { Co }}$ & 228 & 41 & 0.09 & 0.04 & 1.45 \\
CNT $_{\text {ox }}$ Co & 222 & 32 & 0.12 & 0.03 & 1.64 \\
Norit & 1233 & 621 & 0.54 & 0.27 & 0.61 \\
Norit3CoAc & 1147 & 765 & 0.31 & 0.48 & 0.60 \\
Norit3Co & 1050 & 696 & 0.27 & 0.44 & 0.54 \\
B500 & 629 & 550 & 0.22 & 0.16 & 1.25 \\
B5003Co & 619 & 470 & 0.22 & 0.17 & 1.20 \\
\hline
\end{tabular}

$\mathrm{W}_{\mathrm{O}(\mathrm{N} 2)}=$ micropore volume and $\mathrm{V}_{\mathrm{T}}=$ total volume both determined by $\mathrm{N}_{2}$ adsorption. $\mathrm{W}_{\mathrm{O}(\mathrm{CO} 2)}=$ micropore volume determined by $\mathrm{CO}_{2}$ adsorption.

A)

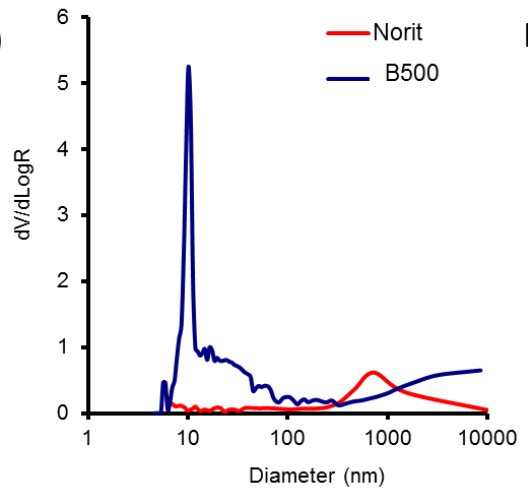

C)

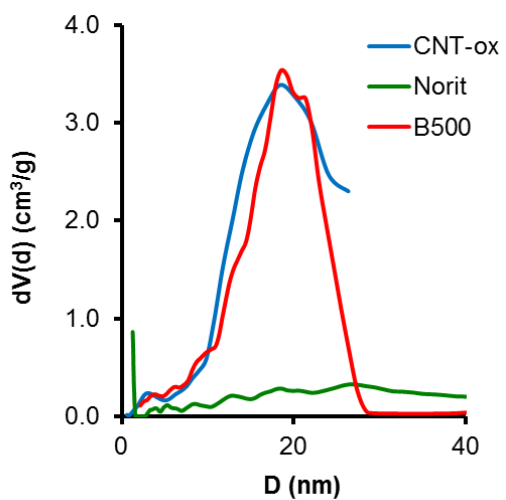

B)

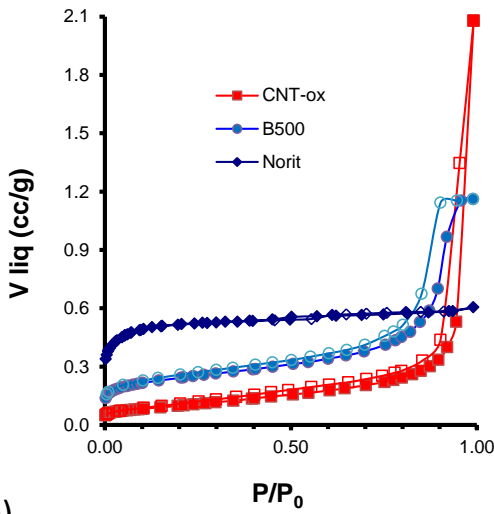

D)

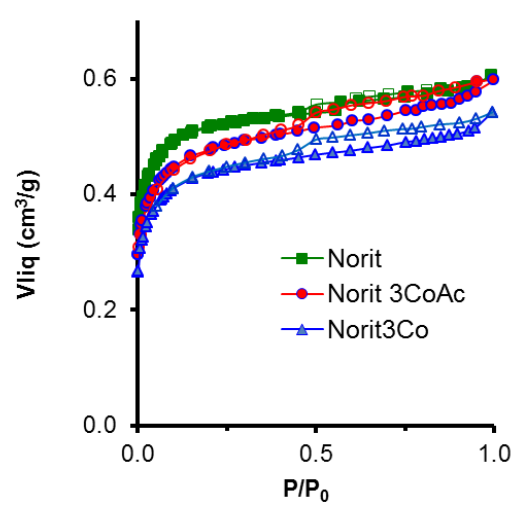

Fig.3 Porous characteristics of supports. A) PSD obtained by mercury porosimetry. B) $\mathrm{N}_{2}$-adsorption isotherms (close symbol: adsorption open symbol: desorption branch). C) PSD obtained from $\mathrm{N}_{2}$-adsorption isotherms by applying the DFT method. D) Influence of the metal precursor on the porosity of catalysts.

The behaviour of B500 as support is clearly intermediate, because this material presents both a well-developed mesoporosity but also a significant microporosity, allowing therefore the deposition of the metal particles on the large external surface of the mesopores and leading to a smaller reduction of the porosity.

The location and distribution of metallic nanoparticles (dispersion) on the different supports were analysed by HRTEM (Fig. 4). In the case of $\mathrm{CNT}_{\mathrm{ox}}$, the internal hollow is around $7 \mathrm{~nm}$ in diameter. Inside this porosity sometimes small Conanoparticles are placed (marked in the Fig. 4 with dense arrows) the most of them being located on the external surface of the CNTs (marked with hollow arrow). The particle size distribution obtained for these samples are showed in Figure 5. Although in general external particles are bigger than those located inside the CNTs, many of them are also quite small but their particle sizes tend to increase with increasing the Co- 
loading thus the distribution shifted from particles with a typical diameter around $7 \mathrm{~nm}$ to particles of around $13 \mathrm{~nm}$, and also the formation of larger particles, exceeding the $20 \mathrm{~nm}$ were observed in this sense, which also favor the CNTs grouping, this fact being related with the detected increase of $W_{0}\left(N_{2}\right)$.
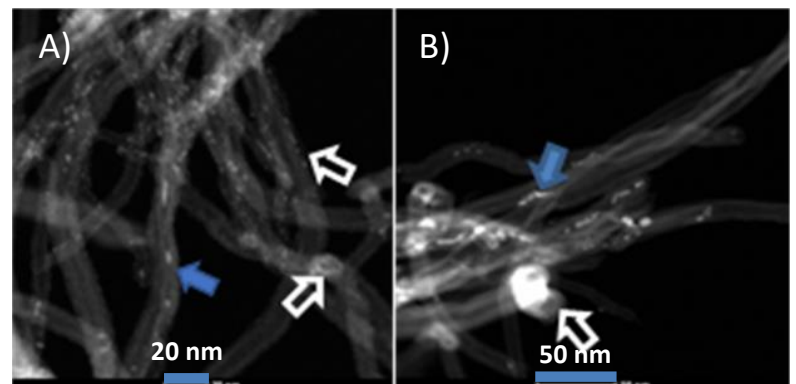

C)
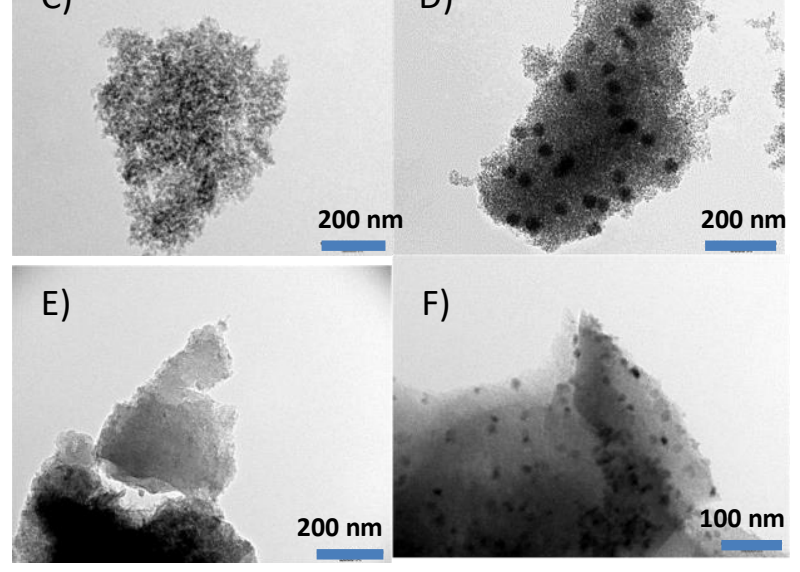

Fig. 4 A) $\mathrm{CNT}_{\text {ox }} 3 \mathrm{Co}$, B) $\mathrm{CNT}_{\text {ox }} 5 \mathrm{Co}$, C) $\mathrm{B} 500-1 \mathrm{Co}$, D) B500-5Co, E) Norit3Co and F) Norit3CoAc samples. Dense arrows point out small CoO-nanoparticles inside the pores whereas hollow arrows indicate those located at external surface.

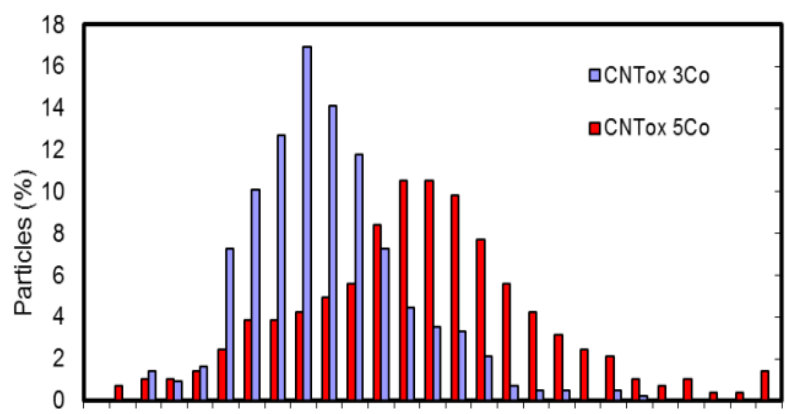

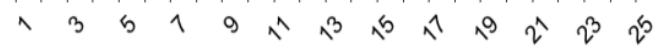

Diameter (nm)

Figure 5. Distribution of the nanoparticle size determined by HTREM for CNTox nCo solid with high

between these

mesopore

volume

primary particles and associated to these mesopores provides an adequate support to develop highly dispersed catalysts, in such a manner that Co-particles are quite difficult to be distinguished at low Co-loading (Fig. 4C) and causing a poor variation of the porous texture. Nevertheless, sintering increases at high Co-loading (Fig. 4D). The Norit AC is a denser but shows large micropores allowing a very high dispersion of Co-nanoparticles. It is important to stress that the Co-particle size increases when use acetate instead nitrate salt (Fig. 4E and 4F). However, even in the worst case, well distributed nanoparticles with diameter of around $13 \mathrm{~nm}$ were obtained.

Similar information is also obtained by mapping of different areas of the catalysts (Fig. 6), the images showing the good and homogeneous dispersion of Co on the CNTs surface.

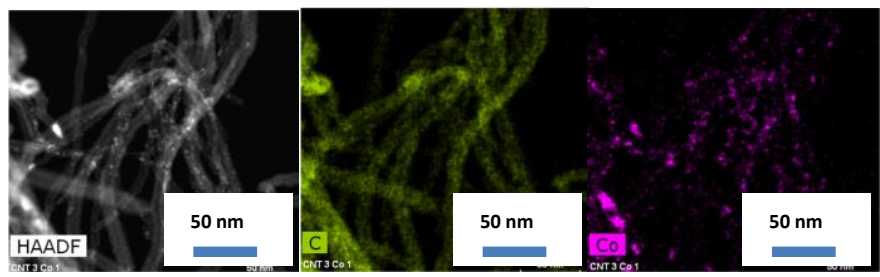

Fig. 6 Mapping of Co-species on the CNTs (CNT ox $3 \mathrm{Co}$ ).

Although the Co-nanoparticles reach a significant size in some samples, their XRD patterns do not present any diffraction peak associated to the Co-phase. At this regard, we try to identify the chemical nature of the metallic particles and the interactions with the supports by XPS analysis of the samples. Thus, the oxygen, carbon and cobalt spectral regions were analysed (Table 3). The peak $\mathrm{C} 1 \mathrm{~s}$ at the carbon region was fitted using four components. The first one at $284.9 \mathrm{eV}$ corresponding to aromatic and aliphatic carbon, while the other ones are associated to the presence of heteroatoms; the component at $286.5 \mathrm{eV}$ corresponding to single $\mathrm{C}-\mathrm{O}$ bonds, the component at $288 \mathrm{eV}$ to $\mathrm{C}=\mathrm{O}$ and finally the carbonate species at around $290 \mathrm{eV}$. The oxygen region $\mathrm{O} 1 \mathrm{~s}$ is fitted using two components, the first one at around $532 \mathrm{eV}$ associated to $\mathrm{C}=\mathrm{O}$ bonds and the second one at $533 \mathrm{eV}$ corresponding to $\mathrm{C}-\mathrm{O}$ bonds. In the catalyst, although this region is fitted also using only two components, clearly the component at low B.E. is favoured after impregnation, due to the contribution of oxygen linked to the metal nanoparticles. Consequently, the oxygen content also significantly increases. The atomic Co-content on surface is $5.8 \%$, corresponding to a $18.4 \% \mathrm{wt}$. This value is clearly higher than the nominal loading (3\%) indicating that the metal is well dispersed only on the high external surface of the support. The analysis of the Co-spectral region is notably more difficult ${ }^{36}$ due to the presence of shake-up satellites together with the $\mathrm{Co}(2 \mathrm{p} 3 / 2)$ and $\mathrm{Co}(2 \mathrm{p} 1 / 2)$ photolines (Fig. 7). The components at $781.9 \mathrm{Co}(2 \mathrm{p} 3 / 2)$ and $797.7 \mathrm{eV} \mathrm{Co}(2 \mathrm{p} 1 / 2)$ with the satellite at $787.1 \mathrm{eV}$ suggest the presence of $\mathrm{Co}^{\prime \prime}$, probably as oxide /hydroxide species. ${ }^{37}$ 


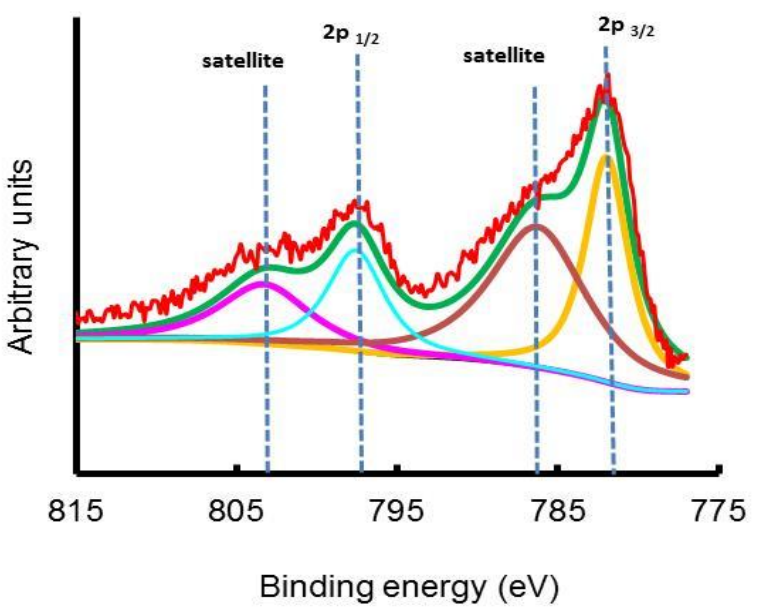

Figure 7 Fitting the XPS spectral region of $\mathrm{Co}$ in the B5003Co catalysts.

The existence of oxidized Co-species on the catalysts surface is also pointed out by TG-DTG experiments carried out in He flow (Fig. 8). Comparing this result with those previously presented for the supports in Fig. 1C, it is noteworthy the new significant peak at around $640 \mathrm{~K}$, that progressively increases with increasing the metal loading. This peak is associated to the WL produced by the reduction of the $\mathrm{Co}$ oxide/hydroxide by the carbon matrix, evolving $\mathrm{CO}_{\mathrm{x}}$.

Interestingly, it is important to remark that these catalysts significantly differ from those previously developed and used in the reaction under study. ${ }^{32,33}$ Previous Co-catalysts were prepared by metal-doping during the sol-gel synthesis of the carbon aerogel, and thereafter carbonized at high temperature ( $1273 \mathrm{~K})$, therefore metal particles were reduced to $\mathrm{Co}^{0}$ during the thermal treatment. In this case, carbon catalysts are prepared by impregnation, metal particles formed exclusively on the support surface during a soft thermal treatment at $573 \mathrm{~K}$. In such a way, amorphous nanoparticles remaining as cobalt oxides/hydroxides are obtained, which should present therefore also a different catalytic performance as showed below.

Figure 8 TG-DTG of $\mathrm{CNT}_{\text {ox }} 1 \mathrm{Co}(---), \mathrm{CNT}_{\text {ox }} 3 \mathrm{Co}(--)$ and $\mathrm{CNT}_{\text {ox }} 5 \mathrm{Co}(\bullet \bullet \bullet$ catalysts in He flow.

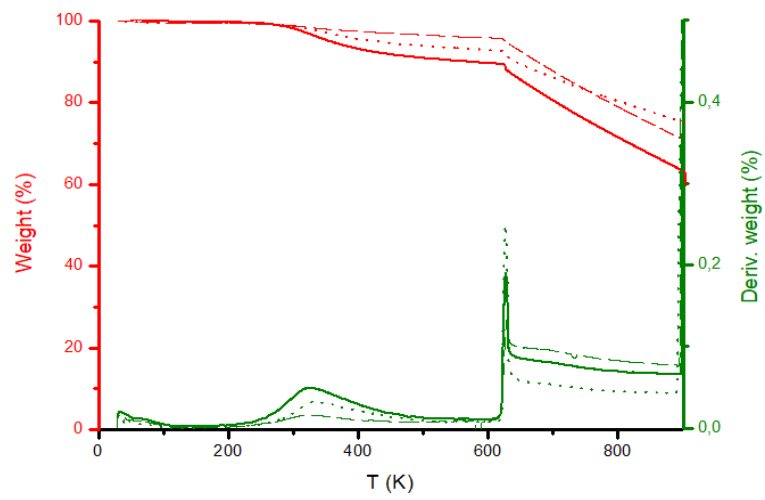

\section{Catalytic performance}

First, the catalytic performance of the investigated nanocarbons used as supports was checked on the condensation of 2-amino-5chlorobenzaldehyde (1a) and ethyl acetoacetate (2) for the synthesis quinolines 3a (Scheme 1) as an analogue of biologically relevant compound. ${ }^{38}$ As demonstrated in our previous studies, the carbon support is active in the reaction when operating at $323 \mathrm{~K}$, producing the quinoline $\mathbf{3 a}$ in low yields probably involving $\pi, \pi$-stacking interactions. $^{21}$

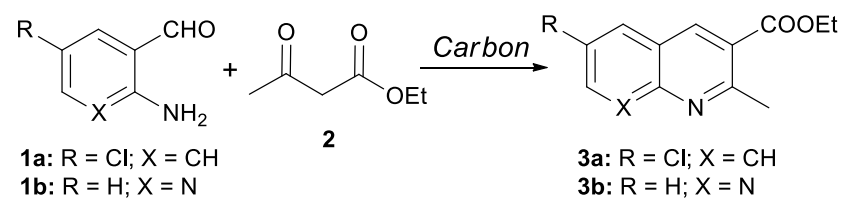

Scheme 1 Friedländer condensation from 2-aminoaryl aldehydes (1) and ethyl acetoacetate (2), under solvent-free conditions.

Fig. 9A depicts the comparison of yield vs time for the carbon supports Norit, B500 and CNT ox when operating at $323 \mathrm{~K}$ exhibiting notable differences at the shortest reaction times. In these cases, the porosity of the samples seems directly affect to the reactivity, the presence of the mesopores in B500 leading to increased yields of compound 3a. Similar trend was observed for the $\mathrm{CNT}_{\text {ox }}$ regarding B500 sample. In this case, the smaller surface can be compensated by large interparticle volume and the presence of OSG generated during the acid treatment, which could be also responsible of the selective formation of quinoline 3 together $\pi, \pi$-stacking interactions between catalysts and reactant as accounted for the previous reported studies concerning the oxygenated carbon materials. ${ }^{21}$ The reaction in the presence of Norit RX3, a mainly microporous sample, led to the quinoline $\mathbf{3 a}$ in notably decreased yields, at the shortest reaction times, due to the diffusion delays into the micropores, but become the most active support at prolonged time on stream probably because this support shows the highest surface area. In Figure 9B, it is shown the yield of quinoline 3 after $4 \mathrm{~h}$ of reaction time for both the uncatalyzed reaction $(12 \%)$ and in the presence of raw CNT (20\%) for comparison purposes.

The reaction under the same experimental conditions used above and in the presence of the Co-supported nanocarbons afforded the quinoline $\mathbf{3 a}$ in almost quantitative yields after $15 \mathrm{~min}$ of reaction time. Then, $\mathrm{CoO}$ nanoparticles supported over the carbon surface notably increased the yields of $\mathbf{3 a}$. Remarkably $\mathrm{CoO}$ is now the catalytic specie, much more active than the $\mathrm{Co}^{0}$ nanoparticles. ${ }^{22}$

Having these results in mind, we carried out the reaction notably increasing the reagent molar ratio (2:5 vs $0.5: 5)$ but also decreasing the reaction temperature, operating at temperature close to room temperature, $303 \mathrm{~K}$. The catalytic behaviour of the samples based on mesoporous nanocarbons by varying the concentration of the active phase is summarized in Fig. 10. For the carbon aerogel-based catalysts, it was observed an increment of yield of $3 a$ as function of the concentration of Co active phase in such a manner that the B5005Co sample led to compound 3a with increased yields. 
Table 3. Atomic Surface composition (by XPS) and Binding Energy of B500 support and B5003Co catalyst.

\begin{tabular}{lllllll}
\hline Sample & C (at\%) & O (at\%) & Co(at\%) & B.E. C (eV) & B.E. O (eV) & B.E. Co (eV) \\
\hline B500 & 92.8 & 7.2 & - & $284.9(74 \%)$ & $531.9(24 \%)$ & -- \\
& & & $286.5(18 \%)$ & $533.8(76 \%)$ & \\
& & & & \\
& & & $288.8(6 \%)$ & & \\
B5003Co & 72.6 & \multirow{2}{*}{21.6} & \multirow{2}{*}{5.8} & $280.8(2 \%)$ & & $781.9(100 \%)$ \\
& & & $286.3(16 \%)$ & $531.5(41 \%)$ & \\
& & & $288.2(6 \%)$ & & \\
& & & & & \\
\end{tabular}
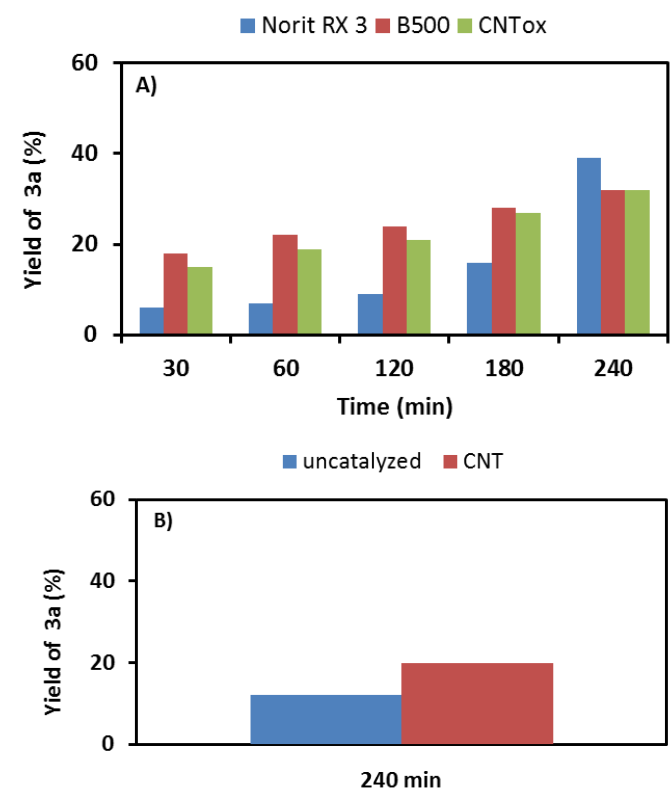

Figure 9 Friedländer condensation from 2-amino-5chlorobenzaldehyde (1a) and ethyl acetoacetate (2) catalysed by nanocarbons, at $323 \mathrm{~K}$, under solvent-free conditions.

However, at short reaction times this catalyst is slower than B5003Co, probably associated to the worse dispersion of the active phase previously showed. This behaviour is even more significant in the $\mathrm{CNT}_{\text {ox }} \mathrm{nCo}$ serie in which it seems that the $\mathrm{CNT}_{\text {ox }} 3 \mathrm{Co}$ nanocarbon shows the optimal amount of $\mathrm{CoO}$ exhibiting the highest yields to product.

Regarding to that, the $\mathrm{CNT}_{\text {ox }} 1 \mathrm{Co}$ and $\mathrm{NTC}_{\mathrm{ox}} 5 \mathrm{Co}$ samples showed a similar catalytic behaviour affording the quinoline $\mathbf{3 a}$ with almost identical yields. In these cases, the highest metal content distributed over the lowest surface area of $\mathrm{CNT}_{\text {ox }}$ also produce the formation of $\mathrm{COO}$ aggregates as demonstrated by the HRTEM images. The effect of the porosity and the nanoparticles location is even more noticeable at intermediate metal loadings (Fig. 10). CoO supported on $\mathrm{B} 500$ are faster than when supported on CNTs, in this case favoured by a high dispersion degree (Fig. 4C). Thus, although B5003Co and CNT ${ }_{0 x} 3$ Co afforded the quinoline 3a in high yields after 1 h of reaction time (up to $60 \%$ ), at short reaction times (15 $\mathrm{min}$ ) B5003Co sample yielded the quinoline $3 a$ in $72 \%$ vs 37 and $16 \%$ for the $\mathrm{CNT}_{\text {ox }} 3 \mathrm{Co}$ and Norit3Co samples, respectively (Fig. 10), this feature demonstrating the advantage of using carbon aerogels.
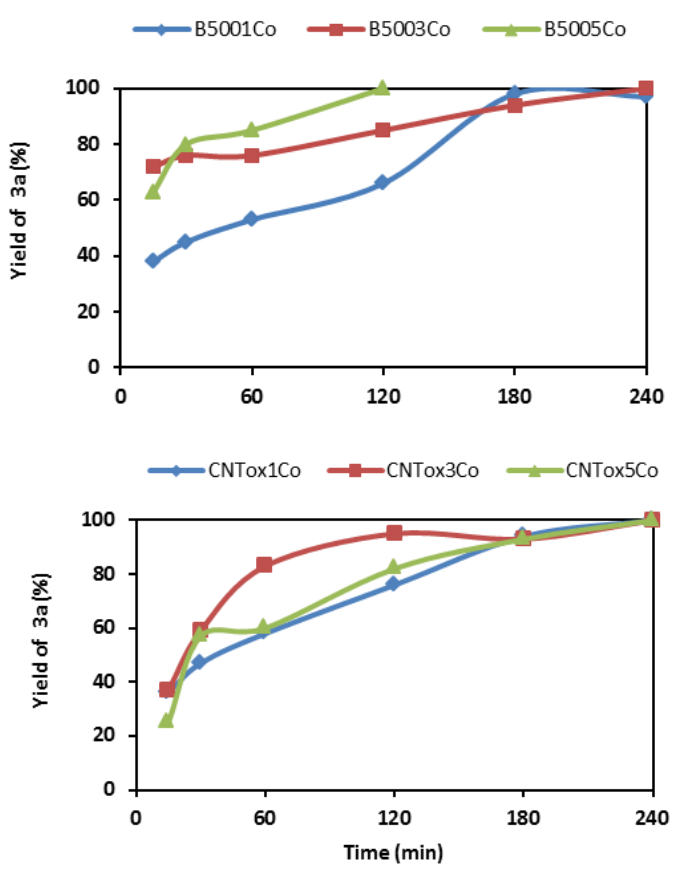

Figure 10 Friedländer condensation from 2-amino-5chlorobenzaldehyde (1a) and ethyl acetoacetate (2) catalysed by CoO-supported nanocarbons -B500nCo and CNT oxnCo series - at 303 $\mathrm{K}$, under solvent-free conditions.

This figure clearly pointed out the importance of the location of the active phase and in turn of the support porosity. Thus, the worse initial performance of $\mathrm{CoO}$ supported on microporous carbon Norit is evidently associated to the delay caused by the diffusion of reactants and products to the catalytic sites, as commented, while the best performance was observed for the highly mesoporous carbon aerogel. The $\mathrm{CNT}_{\text {ox }} \mathrm{nCo}$ serie exhibited an intermediate catalytic behavior, probably due to the Co-nanoparticles distribution on both the internal and external surface of the nanotubes. Conversion increases slowly in the case of B500nCo catalysts with the reaction time because the concentration of the reactants strongly decreases in the first stage. However, in both $\mathrm{CNT}_{\text {ox }} \mathrm{nCo}$ and NoritnCo series, the Co-nanoparticles inside the pores became progressively active as they were located in deeper and narrower porosity. Conversion increased faster in the case of CNTs because the internal diameter is of around $7 \mathrm{~nm}$ (mesopores) regarding the micropores 
present in the Norit support. Nevertheless, this sample became finally the most active due to the higher catalytic surface and high Co-dispersion.

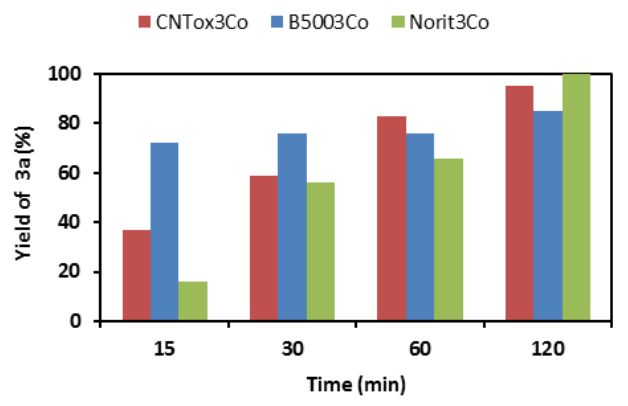

Figure 11 Friedländer condensation from 2-amino-5chlorobenzaldehyde (1a) and ethyl acetoacetate (2) catalysed by CoO-supported nanocarbons - B5003Co, $\mathrm{CNT}_{\text {ox }} 3 \mathrm{Co}$ and Norit3Co - at $303 \mathrm{~K}$, under solvent-free conditions.

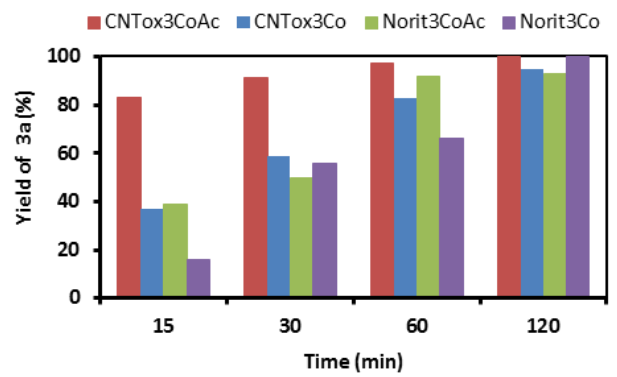

Figure 12 Friedländer condensation from 2-amino-5chlorobenzaldehyde (1a) and ethyl acetoacetate (2) catalysed by CoO-supported nanocarbons - $\mathrm{CNT}_{\mathrm{ox}} 3 \mathrm{CoAc}$ and Norit3CoAc - at 303 $\mathrm{K}$, under solvent-free conditions.

The impregnation salt nature has a deep impact on the location of the Co-nanoparticles as previously commented. The metal dispersion decreases when using $\mathrm{Co}\left(\mathrm{CH}_{3} \mathrm{CO}_{2}\right)_{2}$ instead $\mathrm{Co}\left(\mathrm{NO}_{3}\right)_{2}$ (Fig. 4). When supported on $\mathrm{CNT}_{\text {ox }}$ (Fig. 12) it is observed than in spite the loss of the active site number by the stronger sintering, $\mathrm{CNT}_{\text {ox }} 3 \mathrm{CoAc}$ catalyst is more active than $\mathrm{CNT}_{\text {ox }} 3 \mathrm{Co}$. When use the microporous activated carbon Norit as support, Norit3CoAc is more active than Norit3Co, especially at the shortest reaction times, but the activity of the later improve with the reaction time. This is again related with the accessibility of reactants to the active phase, thus, the large nanoparticles from the acetate precursor are formed progressively out of the microporous system of the support being more active at short reaction time while with increasing this parameter the best performance is observed for the small nanoparticles located inside the pores. Therefore, the used metal precursor in the catalyst synthesis also influences the catalytic performance and the relationship between particle size and location should be therefore optimized to obtain fast, selective and stable catalysts. Finally, remark that $\mathrm{CNT}_{\text {ox }} 3 \mathrm{CoAc}$ was reused during three times with only a slight activity loss (80, 79 and $72 \%$ after $15 \mathrm{~min}$ of reaction time).

In addition, we study the reaction using 2-aminonicotinaldehyde (1b) and ethyl acetoacetate (2) catalyzed by $\mathrm{CNT}_{\text {ox }} 3 \mathrm{CoAc}$ (Scheme 1). Fig. 13 depicts the yield of $\mathbf{3 b}$ vs time in the presence of $\mathrm{CNT}_{\text {ox }} 3 \mathrm{CoAc}$, at $303 \mathrm{~K}$, observing a pronounced decay of yield compared to when using benzaldehyde 1a, as expected according our previous results, that suggested a lower reactivity probably due to the acid-base strong interactions of both reactant $\mathbf{1} \mathbf{b}$ and product $\mathbf{3} \mathbf{b}$ with active catalytic sites. ${ }^{10,20}$

Concerning the reaction mechanism for the investigated condensation, when using $\mathrm{Co}^{0}$ doped carbon aerogels we previously proposed the in situ generation of $\mathrm{Co}^{0}-\mathrm{Co}^{\prime l}$ catalytic system, which would initiate the condensation reaction between reactants. ${ }^{12}$ The enhanced catalytic performance observed for the nanocarbons herein reported is then attributed to the presence of $\mathrm{CoO}$. Based on our experience, $\mathrm{CoO}$ could act as dual catalyst in which $\mathrm{O}$ atom would activate the ethyl acetoacetate, through its enol form, whereas Co increasing the electrophilic character of the carbonyl acceptor strongly favouring the reaction. Subsequent heterocyclization followed by double dehydration steps would yield the corresponding quinoline.

Finally, Table 4 summarizes the catalysts described for the synthesis of quinolines 3 , demonstrating the high potential of $\mathrm{CoO}$ supported carbon catalyst under study. These catalytic materials were found to be easily prepared as a sustainable alternative to metal organic frameworks (MOFs), particularly Cu-BTC, bifunctional mesoporous silicas (AP/AIMCF) and even other basic carbocatalysts with high metal oxide loadings (PET/MAG), showing both superior thermal stability and enhanced catalytic performance.

Table 4. Catalysts reported for the Friedländer reaction from 2-aminoaryl aldehydes (1) and ethyl acetoacetate (2).

\begin{tabular}{|c|c|c|c|c|c|}
\hline Catalyst & Reaction product & Reaction Conditions & $\begin{array}{l}\text { Time } \\
(\min )\end{array}$ & $\begin{array}{c}\text { Yield } \\
(\%)\end{array}$ & Ref. \\
\hline PET/MAG & 3a & 1a $(0.5 \mathrm{mmol}), 2(5 \mathrm{mmol})$, catalyst $(25 \mathrm{mg}), 293 \mathrm{~K}$ & 60 & 71 & 20 \\
\hline RFCoS & 3a & 1a $(0.5 \mathrm{mmol}), 2(5 \mathrm{mmol})$, catalyst $(25 \mathrm{mg}), 323 \mathrm{~K}$ & 60 & 93 & 22 \\
\hline Cu-BTC & $3 a$ & $1 \mathrm{a}(0.5 \mathrm{mmol}), 2(5 \mathrm{mmol})$, catalyst $(25 \mathrm{mg}), 323 \mathrm{~K}$ & 60 & 58 & 22 \\
\hline B5003Co & $3 a$ & 1a (2 mmol), 2 (5mmol), catalyst $(25 \mathrm{mg}), 303 \mathrm{~K}$ & 15 & 71 & This work \\
\hline $\mathrm{CNT}_{\mathrm{O}} 3 \mathrm{CoAc}$ & $3 a$ & & 15 & 86 & This work \\
\hline B5003Co & $3 a$ & 1a $(2 \mathrm{mmol}), 2$ (5mmol), catalyst $(25 \mathrm{mg}), 303 \mathrm{~K}$ & 15 & 73 & This work \\
\hline $\mathrm{CNT}_{\mathrm{ox}} 3 \mathrm{CoAc}$ & 3b & 1b $(2 \mathrm{mmol}), \mathbf{2}(5 \mathrm{mmol})$, catalyst $(25 \mathrm{mg}), 303 \mathrm{~K}$ & 120 & 51 & This work \\
\hline AP/Al-MCF & 3b & 1b $(0.5 \mathrm{mmol}), \mathbf{2}(5 \mathrm{mmol})$, catalyst $(25 \mathrm{mg}), 323 \mathrm{~K}$ & 120 & 42 & 39 \\
\hline
\end{tabular}




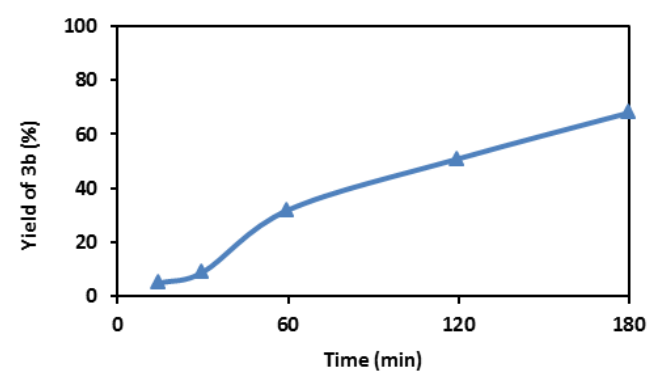

Fig. 13 Friedländer condensation from 2-aminonicotinaldehyde (1b) and ethyl acetoacetate (2) catalyzed by $\mathrm{CNT}_{\text {ox }} 3 \mathrm{CoAc}$, at $303 \mathrm{~K}$, under solvent-free conditions.

\section{Conclusions}

We report herein the first examples of $\mathrm{CoO}$ supported on carbon nanomaterials highly efficient in the synthesis of nitrogen heterocyclic compounds through the Friedländer condensation, under solvent-free and mild conditions. Three series of eco-sustainable catalysts, exhibiting enhanced catalytic performance are prepared and characterized and these properties related with their catalytic performance. The role of the support (AC, CNT or carbon aerogels) porosity and surface chemistry influences the formation of $\mathrm{CoO}$ nanoparticles on different porosity ranges. The size and distribution of these nanoparticles are also influenced by the Co-precursor salt. Sintering increased with increasing the metal loading. In the case of mesoporous aerogels the nanoparticles can be located in the mesoporosity and large micropore range, favouring a homogeneous distribution and the accessibility of reactants (fast reactions). On the contrary, microporous ACs with high specific surface area induce slowly reaction, but yields increased with the reaction time obtaining yields even greater than in the previous case. Using $\mathrm{CNT}_{\text {ox }}$, in spite that present the highest pore volume, some of the metal nanoparticles are formed inside the nanotubes, showing an intermediate behaviour between mesoporous carbon aerogels and microporous ACs. Among the explored samples, B5005Co was found to be the most efficient catalyst providing highest conversions to product, at the shortest reaction times, demonstrating the advantage of using mesoporous carbon aerogels as pure carbon support. The CoO-Carbon catalysts herein reported are found to be much more efficient than MOFs, bifunctional mesoporous silicas and even basic carbon catalysts, showing also a notable stability along different reaction cycles. Then, these interesting results open the doors to the development of functional carbon aerogels able to efficiently catalyse the synthesis of relevant heterocyclic systems through cascade reactions.

\section{Conflicts of interest}

There are no conflicts to declare.

\section{Acknowledgements}

This work has been supported by Spanish Ministry (CTM 201456668-R project).

\section{Notes and references}

1 J.L. Figueiredo, Nanotechnology in Catalysis: Applications in the Chemical Industry, Energy Development, and Environment Protection, in: B. Sels, M. Van de Voorde (Eds.), Application of Nanocarbon Materials to Catalysis, Wiley-VCH Verlag GmbH \& Co, KGaA, 2017, pp. 37-55.

2 P. Serp and J. L. Figueiredo, Carbon Materials for Catalysis, John Wiley \& Sons, Hoboken, NJ, 2009.

3 E. Pérez-Mayoral, V. Calvino-Casilda and E. Soriano, Catal. Sci. Tech., 2016, 6, 1265-1291.

4 F. Rodriguez-Reinoso, Carbon, 1998, 36, 159-175.

5 F. J. Maldonado-Hódar, Catal. Today, 2013, 218, 43-50.

6 Y. Yıldız, I. Esirden, E. Erken, E. Demir, M. Kaya and F. Sen, Chem. Select, 2016, 1, 1695-1701.

7 A. B. Dongil, B. Bachiller-Baeza, I. Rodríguez-Ramos, J. L. G. Fierro and N. Escalona, RSC Adv., 2016, 6, 26658-26667.

8 P. Basu, P. Prakash, E. Gravel, N. Shah, K. Bera, E. Doris and I. N. N. Namboothiri, ChemCatChem, 2016, 8, 1298-1302.

9 J. John, E. Gravel, A. HagÀge, H. Li, T. Gacoin and E. Doris, Angew. Chem. Int. Ed., 2011, 50, 7533-7536.

10 R. Kumar, E. Gravel, A. HagÀge, H. Li, D. V. Jawale, D. Verma, I. N. N. Namboothiri and E. Doris, Nanoscale, 2013, 5, 64916497.

11 D. V. Jawale, E. Gravel, V. Geertsen, H. Li, N. Shah, I. N. N. Namboothiri and E. Doris, ChemCatChem, 2014, 6, 719-723.

12 R. Kumar, E. Gravel, A. HagÀge, H. Li, D. Verma, I. N. N. Namboothiri and E. Doris, ChemCatChem, 2013, 5, 3571-3575.

13 S. Donck, E. Gravel, N. Shah, D. V. Jawale, E. Doris and I. N. N. Namboothiri, RSC Adv., 2015, 5, 50865-50868.

14 N. Shah, E. Gravel, D. V. Jawale, E. Doris and I. N. N. Namboothiri, ChemCatChem, 2014, 6, 2201-2205.

15 N. Shah, E. Gravel, D. V. Jawale, E. Doris and I. N. N. Namboothiri, ChemCatChem, 2015, 7, 57-61.

16 Z. Hasan, Y. S. Ok, J. Rinklebe, Y. F. Tsang, D.-W. Cho and H. Song, J. Alloys Compounds, 2017, 703, 118-124

17 M. Biegun, X. Chen and E. Mijowska, ChemElectroChem 2018, 5, 2681-2685

18 J. Marco-Contelles, E. Pérez-Mayoral, A. Samadi, M. C. Carreiras and E. Soriano, Chem. Rev., 2009, 109, 2652-2671.

19 J. López-Sanz, E. Pérez-Mayoral, E. Soriano, D. OmenatMorán, C. J. Durán, R.M. Martín-Aranda, I. Matos and I. Fonseca, ChemCatChem, 2013, 5, 3736-3742.

20 M. Godino-Ojer, A, J. López Peinado, R. M. Martín Aranda, J. Przepiorski, E. Pérez-Mayoral and E. Soriano, ChemCatChem, 2014, 6, 3440-3447.

21 M. Godino-Ojer, E. Soriano, V. Calvino-Casilda, F. J. Maldonado-Hódar and E. Pérez Mayoral, Chem. Eng. J., 2017, 314, 488-497.

22 M. Godino-Ojer, A. J. López Peinado, F. J. Maldonado-Hódar, and E. Pérez-Mayoral, ChemCatChem, 2017, 9, 1422-1428.

23 M. Godino-Ojer, R. M. Martín-Aranda, F. J. Maldonado-Hódar, A. F. Pérez-Cadenas and E. Pérez-Mayoral, Mol. Catal., 2018, 445, 223-231.

24 M. Trépanier, A. Tavasoli, A. K. Dalai and N. Abatzoglou, Appl. Catal. A, 2009, 353, 193-202.

25 F. J. Maldonado-Hódar, C. Moreno-Castilla, J. Rivera-Utrilla and M. A. Ferro-García, Stud. Surf. Sci. Catal., 2000, 130B, 1007-1012. 
26 M. M. Dubinin, Chemistry and Physics of Carbon, in: P. L. Walker, Jr. (Ed), Marcel Dekker, New York, 1966. Vol. 2, pp. 51-120.

27 R.C. Bansal, J.B. Donnet and H.F. Stoeckli, Active Carbons, Marcel Dekker, New York. 1988.

28 A. V. Neimark, Y. Lina, I. Peter, P. I. Ravikovitch and M. Thommes, Carbon, 2009, 47, 1617-1628.

29 F. Duarte, F. J. Maldonado-Hodar and L. M. Madeira, Appl. Catal. B: Env., 2011, 10, 109-115.

30 F. J. Maldonado-Hódar, M. A. Ferro-García, J. Rivera-Utrilla and C. Moreno-Castilla, Carbon, 1999, 37, 1199-1205.

31 J. L. Figueiredo, M. F. R. Pereira, M. M. A. Freitas and J. J. M. Orfao, Carbon, 1999, 37, $1379-1389$.

32 J. F. Vivo-Vilches, E. Bailón-García, A. F. Pérez-Cadenas, F. Carrasco-Marín and F. J. Maldonado-Hódar, Carbon, 2014, 68, 520-530.

33 Q. H. Yang, P. X. Hou, S. Bai, M. Z. Wang and H. M. Cheng. Chem. Phys. Lett., 2001, 345, 18-24.

34 D. Cazorla-Amoros, J. Alcañiz-Monge, M. A. De la Casa-Lillo and A. Linares-Solano, Langmuir, 1999, 14, 4589-4596.

35 F. M. Duarte, F. J. Maldonado-Hódar and L. M. Madeira, Appl. Catal. A, 2013, 458, 39-47.

36 C. Moreno-Castilla, F. J. Maldonado-Hódar and A. F. PérezCadenas, Langmuir, 2003, 19, 5650-5655.

37 Z. Kónya, J. Kiss, A. Oszkó, A. Siska and I. Kiricsi, Phys. Chem.Chem. Phys., 2001, 3, 155-158.

38 P. Ornaghi, D. Rotili, G. Sbardella, A. Mai and P. Filetici, Biochem. Pharmacol., 2005, 70, 911-917.

39 A. Smuszkiewicz, E. Pérez-Mayoral, E. Soriano, I. Sobczak, M. Ziolek, R. M. Martín-Aranda and A. J. López-Peinado, Catal. Today, 2013, 218-219, 70-75. 